\title{
Sistemática de um fator de qualidade dos eventos científicos
}

\author{
Adilson Luiz Pinto, Moisés Lima Dutra, Douglas Dyllon Jeronimo de Macedo \\ Universidade Federal de Santa Catarina, Departamento de Ciência da Informação. Florianópolis, \\ Brasil.
}

Alexandre Ribas Semeler

Universidade Federal do Rio Grande do Soul, Instituto de Geociências. Porto Alegre, Brasil.

\section{CASE REPORT}

\begin{abstract}
Resumo
Objetivo. Identificar as características e relevância das publicações em eventos, o impacto de citações (índice-h, g e citação/ano) e a geração de um índice de impacto. Método. Metodologicamente o estudo foi aplicado aos 277 docentes que fazem parte dos PPG's em $\mathrm{Cl}$ e foi desenvolvido um software para realizar a filtragem dos dados dos CV Lattes (ProspectorWare) e foi utilizado o Google Acadêmico e Publish or Perish para identificar os índices de impacto. Resultados. Como principais resultados tivemos uma frequência muito elevada para Enancib e ISKO em termos de publicação, porém os eventos que mais foram citados foram Intercom, SBPC e ISKO (todos com mais de 75 citações/ano). Para o índice-g tivemos como destaque Intercom, ISSI, SBPC e Encontros Nacional de Engenharia de Produção (todos com índice superior a 15). E o índice-h tivemos como destaque Intercom e SBPC (com índice superiores a 10). Em relação a sistemática do fator de qualidade destacamos mais uma vez Intercom (fator 68,19), SBPC (fator 15,32), ISSI $(13,55)$ e Encontro Nacional de Engenharia de Produção $(11,45)$. Conclusões. Consideramos relevante destacar que este estudo é um primeiro contato com a quantificação da qualidade dos eventos científicos na Ciência da Informação e que deve sofrer ajuste em um segundo intento, entretanto, ficou evidente que eventos com maiores estruturas e com a preocupação em disponibilização de conteúdo em inglês recebem mais citações.
\end{abstract}

Palavras-chave

Brasil. Ciência da Informação. Eventos científicos. Eventos da Ciência da Informação. Fator de qualidade de eventos científicos. Geração de indicador.

\section{Systems of a quality factor of scientific meetings}

\begin{abstract}
Objetive. Identify the characteristics and relevance of publications in scientific event, the impact of citations ( $h$-index, g-index and citation/year) and the generation of an impact index. Method. The method that applied to investigated was identify the 277 teachers of the Information Science postgraduate schools, and was developed a software to discover the perform and the filtering of the data of the curriculum Lattes (the ProspectorWare), and was used for complementary analysis the Google Scholar and Publish or Perish to identify the impact indexes. Results. The main results we had a very high frequency for scientific events has a National Symposium of Research in Information Science (ENANCIB) and International Society for Knowledge Organization (ISKO) in terms of publication, but the most frequently cited meetings were Intercom, SBPC and ISKO (all with more than 75 citations/year). For the g-index we had as highlight Intercom, ISSI, SBPC and National Encounters of Production Engineering (all with index higher than 15), and for the h-index was Intercom and SBPC (with index higher than 10). In relation to the quality factor system we once again highlight Intercom (factor 68,19), SBPC (factor 15,32), ISSI $(13,55)$ and National Meeting of Production Engineering $(11,45)$. Conclusions. We consider it relevant to highlight that this study is a first contact with the quantification of the quality of the scientific meetings in the Information Science and that it must undergo adjustment in a second attempt, however, it was evident that meeting with larger structures and with the concern in providing content in receive more quotes.
\end{abstract}

Keywords

Brazil. Generation of indicator. Information Science. Meeting of Information Science. Quality factor of scientific meeting. Scientific meeting. 


\section{Introdução}

Os primeiros eventos científicos (Scientific Meetings), no sentido das ciências modernas, podem ter sido realizados concomitantes com a criação da Royal Society na Inglaterra e a Accademia del Cimento na Itália, no Século XVII. A primeira conferência da sociedade inglesa inclui, por exemplo, os experimentos do matemático e físico francês Denis Papin, como o digestor de ossos, dispositivo utilizado para extrair gorduras de ossos em um ambiente de vapor de alta pressão. Em sentido amplo os Scientific Meetings são parte pervasiva e integral das ciências. Eles têm sido um componente fundamental para ciência moderna desde o seu início, são o lócus onde cientistas se reuniam para disseminar o conhecimento entre seus pares. Junto ao crescimento da especialização das ciências os objetivos e o âmbito das reuniões científicas também se tornaram cada vez mais especializados, desde os workshops por convite até congressos nacionais e internacionais com mini-simpósios e muitas sessões paralelas (SODERQVIST; SILVERSTEIN, 1994).

Hoje as Scientific Meetings não só fornecem arenas para os pesquisadores trocarem informações sobre novas teorias e técnicas mas também servem como analogia para se estudar: (i) a formação das disciplinas científicas; (ii) comunicação científica; (iii) os colégios invisíveis, e; (iv) como fonte de estudos métricos da ciência. Enfim, também podem ser vistas como unidades político-retóricas para negociação de tópicos de pesquisa, para a delimitação de territórios cognitivos e para a distribuição do status científico da hierarquia disciplinar das áreas do conhecimento (SÖDERQVIST; SILVERSTEIN, 1994).

Nesse contexto, entende-se que um evento científico (Scientific Meetings) são reuniões de comunidades de pesquisadores que possuem uma área de investigação em comum, esses eventos podem ser locais, regionais, nacionais ou internacionais; são geralmente chamados de congressos, simpósios, encontros ou fóruns. São lugar comum para comunidades de cientistas comunicarem suas descobertas científicas (HAYASHI; GUIMARÃES 2016).

Para Meadows (1999) os eventos são um tipo de comunicação informal da ciência. Os produtos da comunicação científica informal ${ }^{1}$ apresentam-se nos mais diversos formatos ${ }^{2}$, como trabalhos completos ou resumos e expostos oralmente ou por meio pôsteres, a divulgação da produção científica oriunda dos trabalhos apresentados ocorre em anais desses eventos e podem trazer o texto integral ou apenas os resumos das comunicações, também pode ocorrer a divulgação em números especiais de revistas das áreas de especialização do evento (GUIMARÃES; PIUMBATO; HAYASHI, 2014)

O significado que assumem os produtos da comunicação científica em eventos para uma comunidade de pesquisadores é diferente e depende das áreas da ciência. Por exemplo, os anais de congressos para engenheiros e cientistas da computação são de suma relevância e altamente pontuados. Conforme as canadenses Lisée; Larivière e Archambault (2008), em alguns campos, elas podem ter uma vida útil tão longa quanto a da literatura em geral; em alguns outros, eles parecem ter função tradicional e obsolescem mais rápido.

Geralmente a literatura gerada em eventos funciona como algo transitório para geração de artigos em periódicos científicos (NORONHA; POBLACIÓN; CASTILHO, 1995), dependendo da área ou campo do conhecimento. Para esses autores analisar essa transição pode gerar um tipo de indicador que permite verificar quais são os autores permanentes e os transientes, o que permite acompanhar a trajetória dos autores. Para Schubert, Zsindely e Braun (1983) os eventos científicos internacionais representam canais importantes para a comunicação dos resultados da pesquisa. Podem servir como indicadores da atividade científica dos países, particularmente, demostram a natureza aberta ou fechada das comunidades científicas nacionais, bem como a atração e a repulsão entre certos países.

Os eventos científicos são espaços privilegiados para apresentação de trabalhos em andamento e possibilitam a incorporação de novos insights. É nos eventos científicos que surgem os ditos colégios invisíveis ou as redes de colaboração científica. Esse conceito foi formulado por Solla Price (1961) constituindo uma comunidade informal de pesquisadores que podem não estar fisicamente próximos, que são de nacionalidades diferentes, porém estão unidos pelo mesmo objeto da pesquisa. Nessas redes se observa a realização de trocas de experiências e 
conhecimento entre os pares pesquisadores, os quais geralmente são os autores e coautores dos trabalhos apresentados em eventos (HAYASHI; GUIMARÃES 2016).

Os eventos científicos são o principal meio de divulgação para cientistas e pesquisadores. Servem para anunciar projetos de pesquisa em fases iniciais, parciais ou conclusivas da investigação dos grupos ou de um único indivíduo. Eles proporcionam visibilidade interna e externa para os trabalhos científicos, permitem o compartilhamento dos desenvolvimentos das áreas científicas. Além disso, oferecem condições para que as pessoas se conheçam e estabeleçam relações produtivas de trabalho futuro por meio da troca informações (WITTER; SOUZA, 2007).

\subsection{Objetivos}

Este estudo parte do pressuposto de que existe um ativo bem significante nos trabalhos apresentados em eventos e que para algumas áreas é até o veículo de publicação mais expressivo. Entretanto, para campos como a Ciência da Informação quantificar e dar mérito a trabalho apresentado em eventos ainda é uma realidade distante, visto que a $\mathrm{Cl}$ se preocupa prioritariamente com artigos de revistas e livros. O primeiro pela facilidade de mensuração e o segundo por uma luta de prestar homenagem ao árduo trabalho dos autores e editoras.

Por outro lado, em informações extraoficiais, os eventos que pontuam para a Cl são somente Enancib e ISKO, porém os pesos da validação não são revelados ou nem quantificado na avaliação pela Capes (triênios e quadriênio).

Frente a este veículo de publicação, que consideramos de suma importância para os colégios invisíveis, desenvolvemos um sistema que objetiva classificar o impacto dos principais eventos da $\mathrm{Cl}$.

Em busca de saber quais são estes eventos e seus impactos, o estudo tem como objetivos específicos (i) identificar as características das publicações em eventos por: título do evento; idioma de publicação; País da publicação; tipo de publicação (internacional, nacional, regional e local), destino (cidade da publicação); (ii) analisar o impacto das publicações (índice-h, índice-g e citação por ano), e; (iii) modelar um indicador de impacto.

\subsection{Contexto dos Eventos para a Ciência da Informação}

As áreas que pontuam, na atualidade, trabalhos apresentados em evento são poucas (o caso mais conhecido é da Ciência da Computação), porém com o passar dos anos muitas áreas tiveram esta tipologia científica como elemento quantitativo da produção científica, como Turismo (até o estrato de 2007 pontuava) e Engenharia de Produção era informal mas tinha validade no relatório final. Estas são algumas das áreas que pensaram em tentar justificar todo tipo de produção, independentemente de ser branca ou cinzenta.

Em paralelo temos os documentos de áreas em que a Ciência da Informação fez e faz parte. Em conversas informais nos eventos de coordenadores de Programas de Pós-Graduação em Cl, organizado pela Associação Nacional de Pesquisa de Pós-Graduação em Ciência da Informação, sempre é mencionado que os eventos científicos, em especial o Enancib, pontuava até meados da década de 1990 como um evento A1, com pontuação idêntica de uma revista A1. Entretanto, está informação não está registrada em nenhum documento da área ou da Capes.

Em uma análise aprofundada nos documentos de áreas (Biblioteconomia, depois Ciências Sociais Aplicadas e por fim em Comunicação e Informação) conseguimos constatar que alguns documentos tratavam esta informação no relatório de avaliação da Capes. Em uma escala temporal temos o documento de 1995 a 1997 (CAPES, 1997) que não faz nenhuma menção a eventos científicos, provavelmente deveria ser algo que pontuavam somente no relatório final.

No período de 1998 a 2000 também não existe nenhuma menção sobre como pontuar eventos científicos nas áreas de Comunicação e Ciência da Informação (CAPES, 2000) e isso sucedeu no triénio seguinte, 2001 a 2003, onde nem a palavra evento é encontrada no documento (CAPES, 2003). 
Diferentemente, no triênio 2004 a 2006 existe uma sinalização para a pontuação de eventos científicos, porém não fica evidente qual seria o indicador aplicado para tal produção científica.

a) a inclusão, na quantificação da produção de Ciência da Informação, de textos completos publicados em anais de congressos, desde que os mesmos anais tenham sido publicados com ISBN ou ISBD. A decisão, que ainda não gerou qualquer efeito prático na avaliação do triênio 2004/2006, resulta de uma discussão de anos no interior da área de avaliação, e na qual sistematicamente os programas de Ciência da Informação enunciavam sua discordância em relação à desconsideração dessa produção. Esta decisão pressupõe que seja elaborado um Qualis de eventos, por ora inexistente (CAPES, 2007, p. 12).

No triênio seguinte, 2007 à 2009, existe uma sinalização de "considerar, na produção de Ciência da Informação, a participação dos docentes no ENANCIB - Encontro Nacional de Pesquisa em Ciência da Informação" (CAPES, 2009, p. 3) acordado no Fórum de Coordenadores da área, porém mais uma vez não é relatado o peso deste evento em comparação ao estrato de periódicos.

Seguindo os documentos de áreas, no período de 2010 à 2012, temos várias menções a eventos científicos, no qual o primeiro trata de que será aceito publicação em anais de eventos (CAPES, 2013, p. 10; p. 13; p. 26), entretanto existe um item chamado de classificação de eventos que descreve que "a área CSA1 não avalia eventos, no Triênio 2010-2012" (CAPES, 2013, p. 32). Na verdade, as informações deste documento de área não estão claras, visto que hora faz menção de aceitar e outra hora em um item especial relata que não terá pontuação e avaliação para eventos científicos. Acreditamos que os eventos devem ter sido pontuados no relatório final.

Recentemente temos o quadriênio de 2013 a 2016, descreve que em relação...

\begin{abstract}
...à produção científica e inserção internacional, considera-se: produção científica de qualidade internacional (artigos em periódicos científicos, livros e capítulo de livros) diferenciada em relação aos demais programas da área; artigos decorrentes de pesquisa original, publicados em periódico científico nacional ou estrangeiro qualificado como A 1, A2 ou B1, indexado em bases de dados internacionais em proporções notadamente superiores aos dos demais programas da área; participação em redes e grupos de pesquisa consolidados internacionalmente, com resultados de pesquisa expressos em publicações conjuntas; premiações, homenagens, reconhecimento público e honrarias internacionais; participação qualificada em eventos internacionais de notório reconhecimento na condição de conferencista ou coordenador de mesa... (CAPES, 2016, p. 18, Grifo nosso).
\end{abstract}

Mais uma vez não existe qualquer relato da pontuação para esta tipologia científica, nos levando a considerar que os eventos são importantes mas não devem ter um destaque em termos de pontuados explicita; e que a omissão em não revelar quais são estes eventos auxilia a Ciência da Informação em ajustar este detalhe nos seus relatórios ou nas conversas informais com os Coordenadores do Programas de Pós-Graduação em Ciência da Informação.

Mesmo assim, um dado notório no campo da $\mathrm{Cl}$ é que os eventos que pontuam para este campo são Enancib e ISKO, entretanto a origem oficial desta informação nunca é revelada. E em conversa informal sempre é dito que ambos eventos pontuam como um artigo B1, ou seja 70 pontos.

Diante de todo o impasse dos documentos de área apresentados neste tópico, resolvemos criar, em uma escala temporal de 10 anos, um fator de impacto para os eventos, baseados em três tipos de indicadores, que iremos explorar nos tópicos seguintes.

\title{
2 Aspectos metodológicos
}

A proposta é descritiva com natureza quantitativa, sendo desenvolvida a partir de um sistema de extração de dados da plataforma Lattes do CNPq (entre os dias 15 a 17 de junho de 2018), em formato XML, e aplicado no software ProspectorWare na sua versão beta.

O universo da pesquisa foi de 277 docentes que fazem parte de todos os Programas de Pós-Graduação em Ciência da Informação no Brasil (Acadêmicos), sendo distribuídos da seguinte forma: IBICT n=24; UEL n=10; 
UFBA $n=16$; UFC $n=12$; UFF $n=17$; UFMG-CI $n=16$; UFMG-GOC $n=17$; UFPA $n=8$; UFPB $n=23$; UFPE $n=12$; UFSC $n=24$; UFSCar $n=10$; UnB $n=21$; UNESP $n=36$; Unirio $n=10$, e; USP $n=21$.

A análise temporal perfez de 2008 até 2017, no qual a distribuição anual foi de: 2008 n=664 publicações; 2009 $n=472 ; 2010$ n=509; 2011 n=620; 2012 n=651; 2013 n= 723; 2014 n=1591; 2015 n=602; 2016 n=495; 2017 $n=573$, e $n=12$ sem identificação de data, totalizando $n=6912$ trabalhos apresentados em eventos científicos.

Para as análises de geração de um índice de impacto foi utilizado Google Acadêmico para gerar os indicadores de citação. Também utilizamos o software Publish or Perish para conferências finais e comparação dos dados.

\section{Desenvolvimento}

O propósito deste estudo não é desenvolver um Qualis Eventos, mas sim propor uma sistemática em análise dos trabalhos apresentados em eventos, já que 46,83\% das produções dos 277 docentes avaliados estão concentradas nesta tipologia. Em contrapartida dos $30,11 \%$ de artigos de revistas; $4,89 \%$ de livros e $18,17 \%$ de capítulo de livros.

Para a sistemática de um Qualis Eventos teríamos que trabalhar com a totalidade de eventos (1220) e quantificar todos no quesito de impacto; e também gerar para a Comunicação, visto que estamos falando de uma área de concentração na Capes (Comunicação e Informação).

Em conversa informal com representantes da Capes existe um temor pela sistemática de um Qualis Eventos para a Comunicação e Informação, porém também existe uma sinalização de que este tipo de publicação deve ganhar mais destaque nas avaliações quadrienais, por ser um indicativo de alta concentração de publicação dos docentes credenciados nos Programas de Pós-Graduação.

Tabela 1: Distribuição das publicações por PPG's em Ciência da Informação

\begin{tabular}{|c|c|c|c|c|c|c|c|c|}
\hline 을 & 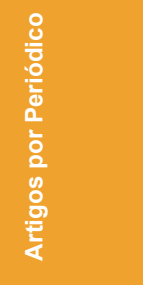 & $\sum_{3}^{0}$ & 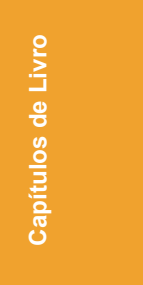 & 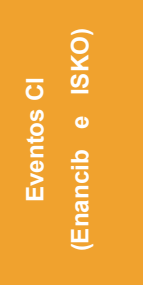 & 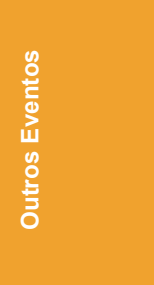 & 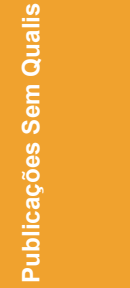 & $\begin{array}{l}\text { 푱 } \\
\text { 음 }\end{array}$ & 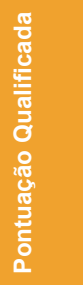 \\
\hline UNESP & $673(18,69)$ & $117(3,25)$ & $706(19,61)$ & $360(10,00)$ & $1054(20,27)$ & $153(4,25)$ & $3063(85,08)$ & 86,00 \\
\hline UFSC & $393(16,37)$ & $40(1,66)$ & $102(4,25)$ & $118(4,91)$ & $329(13,70)$ & $109(4,54)$ & $1091(45,45)$ & 75,30 \\
\hline UFPB & $419(18,21)$ & $72(3,13)$ & $245(10,65)$ & $291(12,65)$ & $394(17,13)$ & $50(2,17)$ & $1471(63,95)$ & 74,90 \\
\hline UNB & $301(14,33)$ & $77(3,66)$ & $181(8,61)$ & $77(3,66)$ & $212(10,09)$ & $46(2,19)$ & $894(42,57)$ & 70,20 \\
\hline UEL & $133(13,30)$ & $17(1,70)$ & $84(8,40)$ & $70(7,00)$ & $266(26,60)$ & $12(1,20)$ & $582(58,20)$ & 68,30 \\
\hline UFMG-GOC & $293(17,23)$ & $21(1,23)$ & $72(4,23)$ & $278(16,35)$ & $322(18,94)$ & $56(3,29)$ & $1042(61,29)$ & 66,70 \\
\hline UFMG-CI & $236(14,75)$ & $60(3,75)$ & $149(9,31)$ & $138(8,62)$ & $171(10,68)$ & $31(1,93)$ & $785(49,06)$ & 66,70 \\
\hline UFPE & $185(15,41)$ & $48(4,00)$ & $132(11,00)$ & $130(10,83)$ & $245(20,41)$ & $27(2,25)$ & $767(63,91)$ & 62,50 \\
\hline UFF & $102(6,00)$ & $31(1,82)$ & $133(7,82)$ & $110(6,47)$ & $140(8,23)$ & $34(2,00)$ & $550(32,35)$ & 61,30 \\
\hline USP & $270(11,21)$ & $52(2,47)$ & $217(10,33)$ & $137(6,52)$ & $333(15,85)$ & $41(1,95)$ & $1050(50,00)$ & 59,40 \\
\hline IBICT & $211(8,79)$ & $57(2,37)$ & $207(8,45)$ & $155(6,45)$ & $217(9,04)$ & $57(2,37)$ & $904(37,66)$ & 54,70 \\
\hline UFBA & $168(10,50)$ & $49(3,06)$ & $109(6,81)$ & $109(6,81)$ & $401(25,06)$ & $27(1,68)$ & $863(53,93)$ & 49,10 \\
\hline UNIRIO & $52(5,20)$ & $17(1,70)$ & $78(7,80)$ & $26(2,60)$ & $78(7,80)$ & $8(0,80)$ & $259(25,90)$ & 35,50 \\
\hline
\end{tabular}




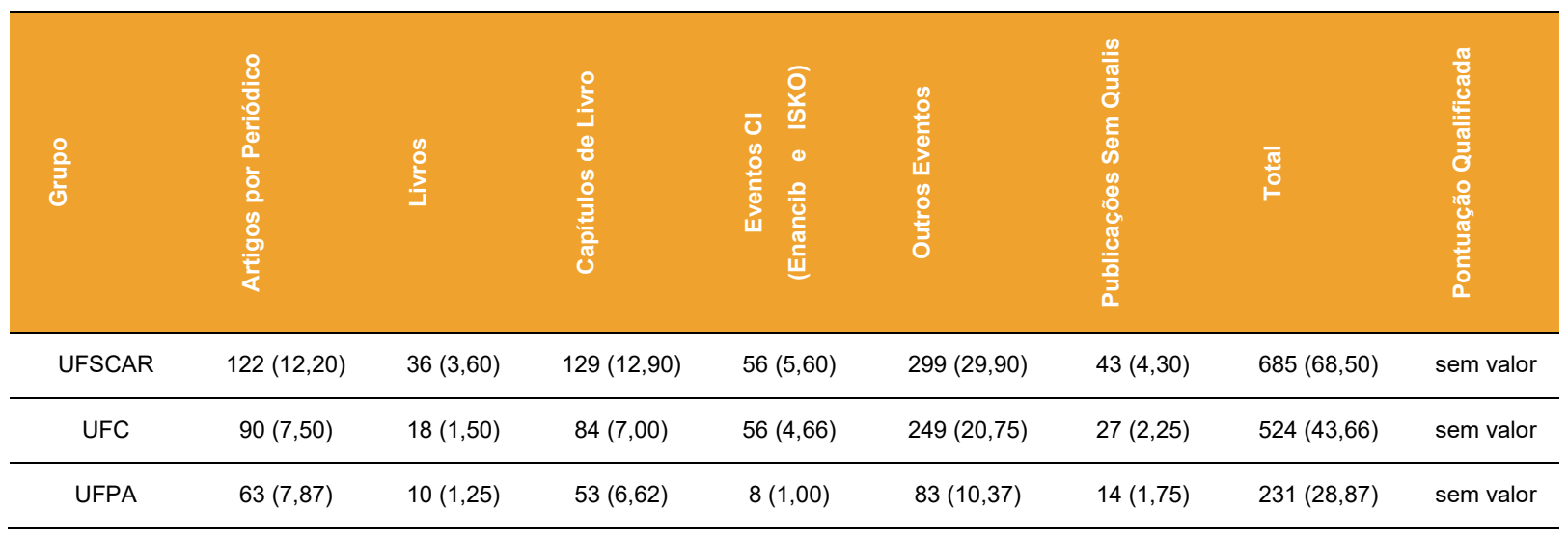

Fonte: Dados da pesquisa. Notas: () são médias de publicação por Programa; Produção Qualificada são dados da Capes 2016, que estão contabilizando somente as 5 principais publicações de cada pesquisador por PPG's.

Deixando de lado uma suposta proposta de Qualis Eventos, nos concentramos em avaliar e analisar o desenvolvimento dos eventos da Ciência da Informação nos últimos 10 anos (2008 até 2017), visando atender a três linhas de frente, que iremos considerar como subtópicos do desenvolvimento deste estudo.

\subsection{Características das publicações em eventos}

Nossa primeira discussão se baseia na representação das publicações em eventos e como as mesmas estão distribuídas em grau de importância para os pesquisadores da Ciência da Informação, no qual foi caracterizada uma tabela para identificar valores primários aos estudos, com quantidade de publicação dos 277 docentes $(n=)$, o título simplificado dos eventos de publicação (Evento Científico), o impacto de publicação índice-h, pelo índiceg e pela citação por ano de cada evento. Desta configuração temos a tabela 2 que está ordenada pelo evento mais buscado na hora de publicação e que conta os valores de seu impacto.

Tabela 2: Dados gerais dos indicadores dos eventos

\begin{tabular}{|c|c|c|c|c|}
\hline$N=$ & Evento Cientifico & $\mathrm{H}$ & G & Citação/ano \\
\hline 2039 & ENANCIB & 4 & 13 & 23,5 \\
\hline 251 & ISKO & 9 & 11 & 77,2 \\
\hline 239 & EDICIC & 6 & 7 & 24,38 \\
\hline 179 & CBBD & 3 & 3 & 1,89 \\
\hline 136 & Congresso de Iniciação Científica da UNESP & 3 & 3 & 5,6 \\
\hline 124 & Seminário em Ciência da Informação & 2 & 3 & 2,22 \\
\hline 123 & SNBU & 4 & 6 & 9,0 \\
\hline 106 & EBBC & 3 & 4 & 4,13 \\
\hline 87 & CINFORM & 2 & 2 & 1,4 \\
\hline 62 & Encontro Internacional Dados, Tecnologia e Informação & 2 & 2 & 1,2 \\
\hline 61 & Encontro de Iniciação Científica & 0 & 0 & 0 \\
\hline 60 & Encontro Internacional de Informação, Conhecimento e Ação & 1 & 1 & 0,25 \\
\hline 52 & Reunião da Linha de Pesquisa Gestão, Mediação e Uso da Informação & 0 & 0 & 0 \\
\hline
\end{tabular}




\begin{tabular}{|c|c|c|c|c|}
\hline $\mathrm{N}=$ & Evento Cientifico & H & G & Citação/anc \\
\hline 48 & Congresso Nacional de Arquivologia (CNA) & 4 & 5 & 3,8 \\
\hline 45 & Encontro de Estudos sobre Tecnologia, Ciência e Gestão da Informação & 0 & 0 & 0 \\
\hline 43 & ISSI & 9 & 23 & 58,5 \\
\hline 43 & Conferência sobre Tecnologia, Cultura e Memória & 2 & 2 & 1,14 \\
\hline 43 & Int Conf on Information Systems \& Technology Management & 1 & 1 & 0,33 \\
\hline 41 & Seminário Científico Arquivologia e Biblioteconomia & 0 & 0 & 0 \\
\hline 41 & Seminário de Pesquisa do PPGCI - Integrando Graduação e Pós-Graduação & 0 & 0 & 0 \\
\hline 39 & ONTOBRAS & 7 & 9 & 23,6 \\
\hline 35 & Enc Direct y Doc de Escuelas de Bibliotecología y Cl del MERCOSUR & 0 & 0 & 0 \\
\hline 32 & EREBD & 0 & 0 & 0 \\
\hline 31 & Congresso Brasileiro de Arquivologia & 3 & 5 & 3,3 \\
\hline 29 & Simpósio Internacional de Iniciação Científica da USP & 2 & 2 & 2,6 \\
\hline 26 & Col em Organização, Acesso e Apropriação da Inf e do Conhecimento & 0 & 0 & 0 \\
\hline 25 & SECIN & 1 & 1 & 1 \\
\hline 24 & ISJO & 0 & 0 & 0 \\
\hline 23 & Colloque International du Réseau MUSSI & 2 & 2 & 0,86 \\
\hline 23 & Encontro de Pesquisadores do Uni-FACEF & 1 & 1 & 0,3 \\
\hline 23 & Conference on Technology, Culture and Memory - СТСM & 1 & 1 & 0,14 \\
\hline 22 & Congresso de Pesquisa Científica & 0 & 0 & 0 \\
\hline 21 & Seminário Hispano-Brasileño de Investigación en Inf, Doc y Sociedad & 0 & 0 & 0 \\
\hline 20 & KM BRASIL & 3 & 3 & 2,9 \\
\hline 19 & Int Conf on Qualitative and Quantitative Methods in Libraries & 2 & 2 & 1,5 \\
\hline 18 & Reunião Brasileira de Ensino e Pesquisa em Arquivologia & 0 & 0 & 0 \\
\hline 17 & CONTECSI & 6 & 6 & 28,9 \\
\hline 17 & Congresso Internacional do Conhecimento e Inovação & 1 & 1 & 0,43 \\
\hline 17 & Encontros Universitários & 0 & 0 & 0 \\
\hline 16 & Congresso de Arquivologia do Mercosul & 1 & 1 & 0,22 \\
\hline 16 & Encontro Anual de Iniciação Científica (EAIC) & 0 & 0 & 0 \\
\hline 16 & Seminário de Pesquisa em Arquivologia da UFBA & 0 & 0 & 0 \\
\hline 15 & Congresso Brasileiro de Ciência da Comunicação & 2 & 3 & 1,44 \\
\hline 15 & Encontro Nacional de Estudantes de Arquivologia & 0 & 0 & 0 \\
\hline 15 & Workshop de Pesquisa em Ciência da Informação & 0 & 0 & 0 \\
\hline 14 & SBPC & 11 & 18 & 84,5 \\
\hline 14 & Semana CRIDI de Pesq e Ext em Cultura, Representação e Inf Digitais & 0 & 0 & 0 \\
\hline 13 & Encontro de Pesquisa em Informação e Mediação - EPIM & 2 & 3 & 2 \\
\hline 13 & Colóquio de Dados, Metadados e Web Semântica (CDMWS) & 0 & 0 & 0 \\
\hline
\end{tabular}




\begin{tabular}{|c|c|c|c|c|c|}
\hline $\mathrm{N}=$ & Evento Cientifico & & H & G & Citação/ano \\
\hline 13 & Semana de Iniciação Científica & & 0 & 0 & 0 \\
\hline 13 & Seminário de Iniciação Científica da UFSC & & 0 & 0 & 0 \\
\hline 12 & International Conference on Science and Technology Indicators (STI) & & 7 & 8 & 15,3 \\
\hline 12 & Seminário de Pesquisa em Ciências Humanas - SEPECH & & 4 & 5 & 5,3 \\
\hline 12 & Encontro Internacional de Catalogadores & & 2 & 3 & 3 \\
\hline 12 & Conferência da SUV & & 1 & 1 & 0,22 \\
\hline 11 & Encontro Nacional de Engenharia de Produção & & 9 & 16 & 71,0 \\
\hline 11 & Congresso Brasileiro de Informática em Saúde & & 5 & 6 & 13,0 \\
\hline 11 & SIICUSP & & 3 & 3 & 4,7 \\
\hline 11 & Congresso de Extensão Universitária da UNESP & & 1 & 1 & 1,3 \\
\hline 11 & Encontro de Pesquisa e Pós-Graduação & & 0 & 0 & 0 \\
\hline 11 & EVINCI - Evento de Iniciação Científica & & 0 & 0 & 0 \\
\hline 11 & Seminário Científico: Arq e Bibliot - Desafios e perspectivas de interlocução & & 0 & 0 & 0 \\
\hline 11 & Seminário Hispano-Brasileiro de Pesq em Infor, Documentação e Sociedade & & 0 & 0 & 0 \\
\hline 10 & INTERCOM & & 15 & 27 & 252,0 \\
\hline 10 & Simpósio Nacional de História & & 6 & 9 & 28,3 \\
\hline 10 & Congresso de Ciências da Comunicação na Região Nordeste & & 4 & 5 & 4,7 \\
\hline 10 & Encontros Universitários da UFC XXXVI & & 1 & 1 & 4 \\
\hline 10 & Colóquio Internacional 'A Medicina na Era da Informação & & 2 & 2 & 0,71 \\
\hline 10 & Col mediações e uso de saberes e da informação: um diálogo França/Brasil & & 0 & 0 & 0 \\
\hline 10 & Cong de Pesquisa cientifica: Inovação, Sustentabilidade, ética e Cidadania & & 0 & 0 & 0 \\
\hline 10 & Seminário Ibero-americano: Arquitetura e Informação & & 0 & 0 & 0 \\
\hline 108 & 12 EVENTOS COM & $\mathrm{N}=9 \mathrm{CADA}$ & 0 & 0 & 0 \\
\hline 152 & 19 EVENTOS COM & $\mathrm{N}=8 \mathrm{CADA}$ & 0 & 0 & 0 \\
\hline 105 & 15 EVENTOS COM & $\mathrm{N}=7$ CADA & 0 & 0 & 0 \\
\hline 144 & 24 EVENTOS COM & $\mathrm{N}=6 \mathrm{CADA}$ & 0 & 0 & 0 \\
\hline 95 & 19 EVENTOS COM & $\mathrm{N}=5$ CADA & 0 & 0 & 0 \\
\hline 256 & 64 EVENTOS COM & $\mathrm{N}=4 \mathrm{CADA}$ & 0 & 0 & 0 \\
\hline 255 & 85 EVENTOS COM & $\mathrm{N}=3$ CADA & 0 & 0 & 0 \\
\hline 426 & 213 EVENTOS COM & $\mathrm{N}=2 \mathrm{CADA}$ & 0 & 0 & 0 \\
\hline 698 & 698 EVENTOS COM & $\mathrm{N}=1 \mathrm{CADA}$ & 0 & 0 & 0 \\
\hline 6912 & 1220 Eventos & registrados & - & - & - \\
\hline
\end{tabular}

Fonte: Dados da pesquisa

O evidente do cenário de impacto dos eventos é que o destaque para os internacionais ficaram evidentes, como o caso International Society for Scientometrics and Informetrics (com fator 13,55), seguido por ISKO (fator 8,58 ), 
CONTECSI - Congresso Internacional de Gestão da Tecnologia e sistemas de Informação (fator 1,79), EDICIC (fator 1,77) e International Conference on Science and Technology Indicators (fator 1,29). É relevante fazer uma ressalva ao ISKO. Este evento tem várias edições (Mundial, Iberoamericana, Francesa e Brasileira), porém contabilizamos todas, em função de ser um evento de âmbito internacional e por ser gestionado por uma Associação Internacional.

Um dado curioso são os eventos de característica nacional na amostra, refletindo que o impacto do evento pouco importa se sua natureza é Internacional, Nacional, Regional ou Local, mas sim a repercussão que, o mesmo, agrega a área ou campo de atuação.

$\mathrm{Na}$ nossa amostra temos alguns eventos nacionais com relativo destaque, como: INTERCOM (fator de 68,19, poderia ser equiparado a muitas revistas A1 da área de Comunicação e Informação), SBPC (fator 15,32), Encontro Nacional de Engenharia de Produção (fator 11,45), ENANCIB (fator 3,10), Simpósio Nacional de História (fator de 2,61) e ONTOBRAS (fator 2,19).

Em se tratando de eventos Regionais e Locais o impacto foi pouco expressivo e não podemos dizer que tiveram relevante destaque na análise, porém vale salientar que o propósito do estudo é que os mesmos passem a ser monitorados sobre a sua frequência, disponibilidade de informações na rede (como anais dos eventos) e o impacto das publicações em termos de citação e até mesmo em indicadores alternativos (acesso, download, luminosidade, menção web, fator de impacto, co-ocorrências entre outros).

\section{Considerações finais}

A sistemática proposta, que em um futuro pode ser algo de qualificação para a $\mathrm{Cl}$ e também para a Comunicação, pode ser útil para identificarmos o esforço que desprende a Ciência da Informação em suas publicações, representando quase $50 \%$ de toda a produtividade do universo de pesquisadores estudados, que são os mais qualificados por serem de Programas de Pós-Graduação.

Respondendo as nossa objetividades de estudo é relevante destacar que a grande representação do Enancib e do ISKO na produtividade se dá pelo fato de que, mesmo informalmente, são os eventos que pontuam para a $\mathrm{Cl}$ e que boa parte dos recursos dos Programas de Pós-Graduação são para custear a ida de seus pesquisadores, em alguns casos os discentes também, aos referidos eventos.

Outro detalhe é que um dos eventos (Enancib) tem uma amplitude de diversidade, concentrando 11 grupos de trabalhos e de certa forma é o evento nacional da Ciência da Informação, então é visível o envolvimento dos pesquisadores do PPG's nesta jornada científica. Entretanto, o ISKO podemos considerar como sendo um evento estratégico de um grupo de trabalho que conseguiu emplacar nas qualificações da $\mathrm{Cl}$. Fato que se deve a gestões de membros deste grupo em agências de fomentos, sendo mais fácil propor tal evento com pontuação.

Os eventos internacionais, por estarem associados a entidades científicas conseguem ter uma representação maior, em função de aplicabilidade pelo idioma e também por uma gestão mais assertiva do site e dos anais dos eventos, quase sempre em idiomas inglês e/ou espanhol.

O impacto de índices como o h, g e citação por ano tiveram destaque para os eventos que são bem amplos e que concentram várias áreas do conhecimento em um só eventos, como foi o caso do Intercom, SBPC, Encontro Nacional de Engenharia de Produção e Ontobras, porém também tivemos um destaque considerável para eventos que disponibilizaram seus conteúdos em inglês, e que nem sempre são anuais, como o caso do ISSI (Estudos métricos), ISKO e o EDICIC (que acontecem em várias edições pelo mundo), o CONTECSI (que é brasileiro, mas que tem um time de evento internacional e com repercussão bem aceita fora do Brasil).

Vale destacar que as citações por ano foram o grande diferencial deste objetivo, no qual somente sete eventos obtiveram um percentual superior a 25 citações ao ano, sendo que o Intercom consegue atingir o incrível índice de 252 citações ao ano. Seria o ideal para qualquer evento no campo da Ciência da Informação, porém para isso seria necessário uma série de medidas para alavancar este indicador, como publicar os resumos inglês, criar DOI para cada uma trabalhos aceitos, gerar anais de eventos (visto que nem todos fornecem isso), edições especiais em revistas de peso entre outras ações de menções aos trabalhos apresentados. 
Como consequência temos a modelagem de um indicador para mensurar a importância de determinados eventos, que buscou objetivar na forma de gerar este identificador por três índices de impacto, contando com a união dos valores do índice-h e do índice-g, potenciados pela citação/ano, gerando o fator da amostra dos eventos analisados.

O relevante deste fator é que ele é muito mais complexo que os parâmetros que se utilizam para gerar os estratos Qualis da Capes de Periódicos. A sua complexidade não se encontra em uma simples indexação, como é o caso das revistas, onde para ser A1 em Comunicação e Informação basta estar na Web of Science e ser indexado em Information Science and Library Science; para ser A2 deve estar indexado na Scopus ou SciELO; e para ser B1 basta estar catalogado no Latindex, Redalyc ou Dialnet.

De verdade os parâmetros para as revistas são muito simplistas, o que estamos propondo neste estudo é um índice de fator preocupado com a visibilidade e com a demanda das citações dos referidos eventos, e por isso consideramos de grande relevância seu aprofundamento em estudos futuros na área de Comunicação e Informação.

Para uma melhora deste tipo de índice podemos futuramente pensar em mais elementos que possam ajuda na sua classificação, como uma visão heurísticas com $\mathrm{N}$ naturezas, como a criação de novos os critérios e uma base para ser inserido outros cálculos, como a periodicidade do evento, se tem patrocínio dos órgãos de fomente (Capes, CNPq, Fapesp entre outros), sua tipologia (como por exemplo ser Workshop, Simpósio, Congresso e/ou Encontro), se tem informado a taxa de aceitação do evento, se tem disposto em quantos dias o evento é apresentado, bem como a distribuição de apresentações e por fim, se o comitê científico é de pesquisadores reconhecimento.

\section{Referências}

CAPES. Documento de área 1998-2000: Comunicação/Ciência da Informação. Brasília: Capes, 2000. Disponível em: <https://www.capes.gov.br/images/stories/download/avaliacao/2000 031 Doc Area.pdf>. Acesso em: 17 jul. 2018.

CAPES. Documento de área 2001-2003: Comunicação/Ciência da Informação. Brasília: Capes, 2003. Disponível em: <https://www.capes.gov.br/images/stories/download/avaliacao/2003 031 Doc Area.pdf>. Acesso em: 17 jul. 2018.

CAPES. Documento de área 2004-2006: Comunicação, Ciência da Informação e Museologia. Brasília: Capes, 2007. Disponível <https://www.capes.gov.br/images/stories/download/avaliacaotrienal/doc areas trienal 2007/2007 Ciencias Sociais Aplicl A val2004-2006.pdf>. Acesso em: 17 jul. 2018.

CAPES. Documento de área 2007-2009: Ciências Sociais Aplicadas I. Brasília: Capes, 2009. Disponível em: <http://www.capes.gov.br/images/stories/download/avaliacao/SOC APLIC 07mai10.pdf>. Acesso em: 17 jul. 2018.

CAPES. Documento de área 2010-2012: Ciências Sociais Aplicadas I. Brasília: Capes, 2013. Disponível em: $<$ https://docs.google.com/viewer?a=v\&pid=sites\&srcid=Y2FwZXMuZ292LmJyfHRyaWVuYWwtMjAxM3xneDoyNDE0MzgzYWU 5YjU3NGQ3>. Acesso em: 17 jul. 2018.

CAPES. Documento de área 2013-2016: Ciências Sociais Aplicadas I. Brasília: Capes, 2016. Disponível em: <http://capes.gov.br/images/documentos/Documentos de area 2017/31 CSA I docarea 2016.pdf>. Acesso em: 17 jul. 2018.

CAPES. Documentos: Biblioteconomia. InfoCapes: Boletim Informativo, v. 5, n. 4, p. 26-29, 1997. Disponível em: <https://www.capes.gov.br/images/stories/download/bolsas/Info4 97.pdf>. Acesso em: 17 jul. 2018.

GUIMARÃES, V; HAYASHI, M. Os eventos científicos: espaços privilegiados para a comunicação da Ciência. Comunicologia, v. 7, n. 2, p. 204-229, 2014. Disponível em: < https://portalrevistas.ucb.br/index.php/RCEUCB/article/view/5656>. Acesso em: 10 jun. 2018.

HAYASHI, M.; GUIMARÃES, V. A comunicação da ciência em eventos científicos na visão de pesquisadores. EmQuestão, v. 22, n. 3, 2016, p. 161-183. Disponível em: <http://dx.doi.org/10.19132/1808-5245223.161-183> Acesso em: 20 mar. 2018.

LISÉE, C.; LARIVIĖRE, V.; ARCHAMBAULT, É. Conference proceedings as a source of scientific information: a bibliometric analysis. Journal of the American Society for Information Science and Technology, v. 59, n. 11, p. 1776-1784, 2008. Disponível em: <http://onlinelibrary. wiley.com/doi/10.1002/asi.20888/abstract>. Acesso em: 3 jan. 2018.

MEADOWS, A. J. A comunicação científica. Brasília: Briquet de Lemos, 1999. 
NORONHA, D. P.; POBLACIÓN, D. A.; CASTILHO, V. Literatura "cinzenta" versus literatura "branca": transição dos autores das comunicações nos snbus para produtores de artigos. Anais... II Encontro Nacional de Pesquisa em Ciência da Informação, 1995. Disponível em: <http://enancib.ibict.br/index.php/enancib/iienancib/paper/viewFile/1314/522>. Acesso em: 22 dez. 2017.

PRICE, D. J. de S. Science Since Babylon. New Haven: Yale University Press, 1961.

SCHUBERT, A.; ZSINDELY, S.; BRAUN, T. Scientometric analysis of attendance at International Scientific Meetings. Scientometrics, v. 5, n. 3, p. 177-187, 1983. Disponível em: <https://doi.org/10.1007/BF02095627>. Acesso em: 1 fev. 2018.

SÖDERQVIST, T.; SILVERSTEIN, A. Participation in scientific meetings: a new prosopographical approach to the disciplinary history of science: the case of immunology, 1951-1972. In: SCANLON, Eillen; HILL, Roger; JUNKER, Kirk. Communicating science: professional contexts. London: Routdlege, 1994. Disponível em: <http://www.sciencedirect.com/science/article/pii/S0008874984712536?via\%3Dihub>. Acesso em: 18 out. 2017.

WITTER, G.; SOUZA, J. British Psychophysiology Society Annual Meeting (2005): análise da produção. Ciência da Informação, v. 36, n. 2, p. 85-91, 2007. Disponível em: <http://revista.ibict.br/index.php/ciinf/article/view/898/722>. Acesso em: 29 jun. 2018. 


\section{Dados dos autores}

Adilson Luiz Pinto

Professor Associado II e Coordenador do Programa de Pós-Graduação em Ciência da Informação da Universidade Federal de Santa Catarina (PGCIN/UFSC). Linhas de pesquisa em Estudos Métricos da Informação, Análise de Redes Sociais e Visualização da Informação.

adilson.pinto@ufsc.br

\section{Moisés Lima Dutra}

Professor do Programa de Pós-Graduação em Ciência da Informação da Universidade Federal de Santa Catarina (PGCIN/UFSC). Linhas de pesquisa em Big Data, Web Semântica e Ciência de Dados.

moises.dutra@ufsc.br

\section{Douglas Dyllon Jeronimo de Macedo}

Professor do Programa de Pós-Graduação em Ciência da Informação da Universidade Federal de Santa Catarina (PGCIN/UFSC). Linhas de pesquisa em Redes de Computadores, Bancos de Dados NãoConvencionais, Sistemas Distribuídos.

douglas.macedo@ufsc.br

\section{Alexandre Ribas Semeler}

Bibliotecário de Dados do Instituto de Geociências da Universidade Federal do Rio Grande do Sul. Pósdoutorando do PGCIN/UFSC. Linhas de pesquisa em e-Sciences, Data Sciences, Data Librarianship. alexandre.semeler@ufrgs.br

Received - Recebido: 2018-08-20

Accepted - Aceitado: 2019-07-22

\section{Notas}

\footnotetext{
${ }^{1}$ Uma comunicação informal é em geral efêmera, sendo posta à disposição apenas de um público limitado. A maior parte da informação falada é, portanto, informal, do mesmo modo que a maioria das cartas pessoais. (MEADOWS, 1999, p. 7)

${ }^{2}$ TCNP - trabalho com resultados completos (ou quase) de pesquisa, ainda não publicado como artigo de periódico; TRP trabalhos com resultados parciais de pesquisas em andamento; TCP - trabalho com resultados completos de pesquisa, já publicado como artigo de periódico; LP - linhas de pesquisa de seu grupo; PP - proposta de projeto de pesquisa (HAYASHI; GUIMARÃES 2016, p.171).
}

\section{$(\mathrm{cc}) \overline{\mathrm{BY}}$}

This work is licensed under a Creative Commons Attribution 4.0

United States License.

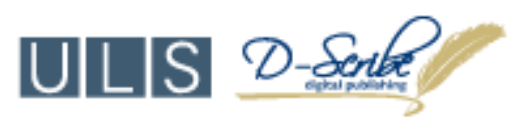

This journal is published by the University Library System of the University of Pittsburgh as part of its D-Scribe Digital Publishing Program and is cosponsored by the University of Pittsburgh Press. 